\title{
Flooding in the Mississippi River Basin in Minnesota, Spring 2001
}

\section{INTRODUCTION}

During spring 2001 there was much flooding in the Mississippi River Basin in Minnesota. Greater than normal precipitation starting with late fall rains in 2000, greater than normal snowfalls, a delayed snowmelt, and record rains in April, all contributed to the flooding. Parts of the southern one-half of Minnesota had streamflows of magnitudes not seen in more than 30 years. Approximately 50 counties were declared disaster areas with greater than 34 million dollars in total reported flood damage (S. Neudahl,
Department of Public Safety, Division of Emergency Management, oral commun. July 9, 2001).

Record flows were recorded at nine U.S. Geological Survey (USGS) continuous-streamflow gaging stations within Minnesota or along the Wisconsin border. In addition, 14 stations recorded their 2nd or 3rd highest peaks of record. These gaging stations are maintained by the USGS as part of a network of gages used by the USGS, U.S. Army Corps of Engineers, National Weather Service, Minnesota Department of Natural

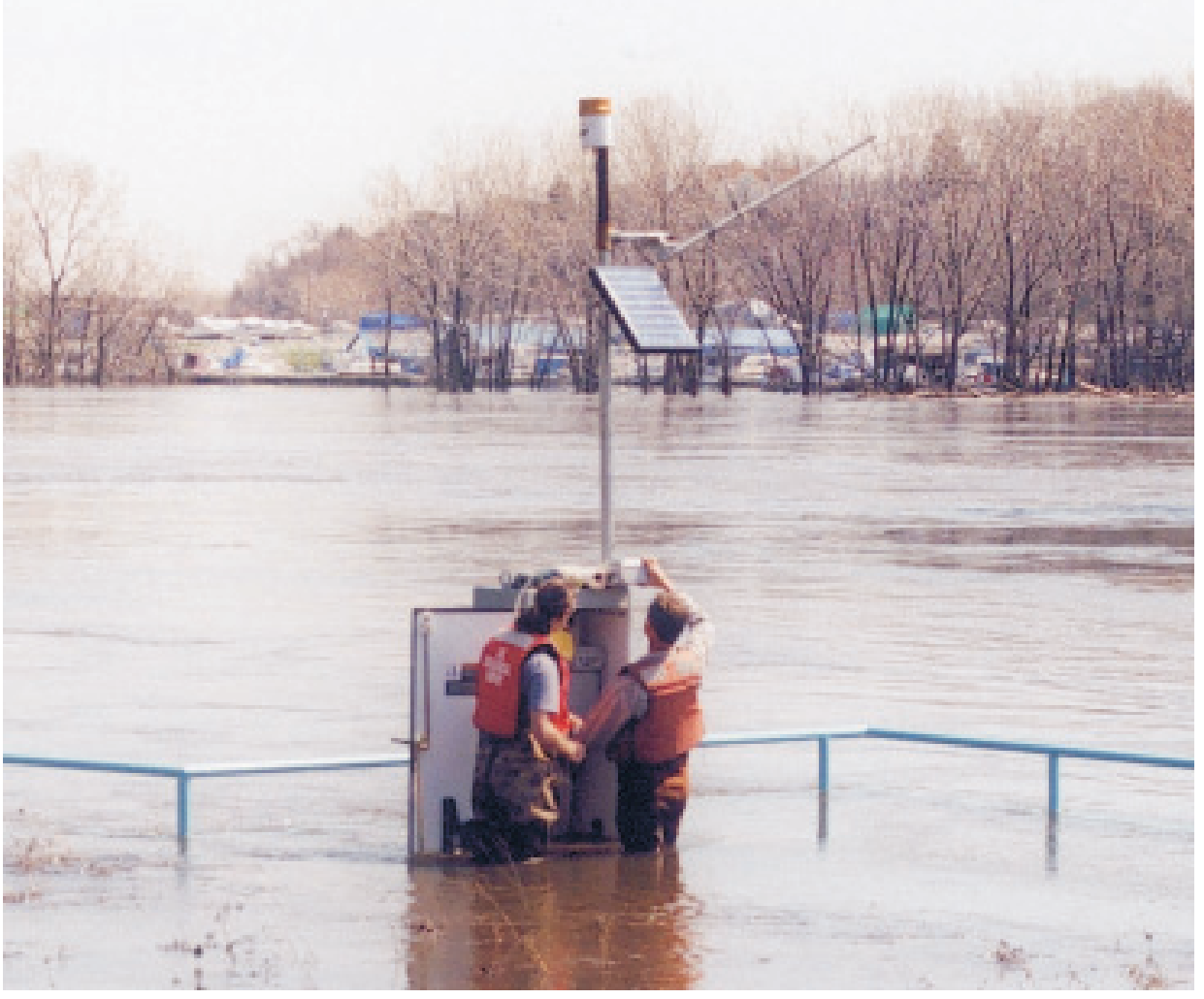

Mississippi River at St. Paul, Minnesota, April 23, 2001. Retrofitting a monitoring station for high water. (U.S. Geological Survey)
Resources, as well as other State and local agencies. Stage and discharge data gathered at these gages are used for flood forecasting, operations of dams and diversions, managing emergency activities during floods, and performing follow-up studies to better understand river floodflow characteristics. This report summarizes flooding in that part of the Mississippi River Basin that includes stream-gaging stations within the borders of Minnesota and monitoring points along the Minnesota-Wisconsin border.

Peak stages and discharges are included in this report for 44 streamgaging stations (table 1) maintained by the USGS in the Mississippi River Basin. Stage, in feet, is based on an arbitrary scale established when a gaging station is installed. Discharges are instantaneous determinations of peak flow, in cubic feet per second. This peak flow information is ranked with the five greatest discharges of record for each station. In addition, floodrecurrence intervals are included for stations with at least 20 years of record, and/or with little influence on peak flows by regulation. Flood-recurrence intervals are expressed in years and are the probability of a flood of this magnitude occurring. A 25-year event can be expected to occur, on average, once every 25 years. This probability is independent of individual events, so two "25-year" floods can occur in successive years. It is also possible for the probability value assigned to a flood (for example, greater than 25-year event) to change over time. As peak flows are included from subsequent years, flood frequencies previously determined can change 
Table 1. Provisional flood information on major Minnesota streams in the Mississippi River Basin.

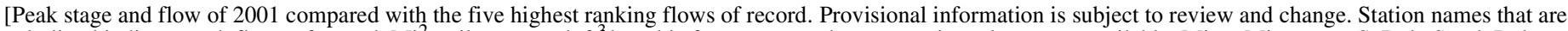

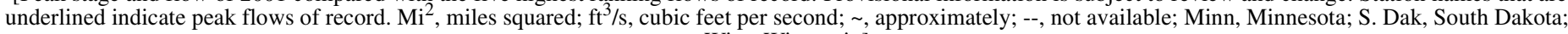

\begin{tabular}{cc}
\hline Station & U.S. Geological Survey gaging station and \\
number & (downstream order number) \\
(Figure 1) & \\
\hline
\end{tabular}
Wisc, Wisconsin]

\begin{tabular}{|c|c|c|c|c|c|c|c|}
\hline \multicolumn{8}{|c|}{ Mississippi River Basin } \\
\hline \multirow[t]{6}{*}{1} & Mississippi River at Grand Rapids, Minn. & 3,370 & 118 & Sept. 3, 1948 & ${ }^{1} 15.20$ & 12,500 & -- \\
\hline & $(05211000)$ & & & April 17, 1969 & -- & 24,610 & -- \\
\hline & & & & May 29, 1950 & -- & 4,160 & -- \\
\hline & & & & April 16, 1945 & -- & 4,070 & -- \\
\hline & & & & Aug. 21, 1999 & -- & 4,060 & -- \\
\hline & & & & Apr. 18, 2001 & 10.06 & ${ }^{3} 2,740$ & -- \\
\hline \multirow[t]{5}{*}{2} & Mississippi River at Aitkin, Minn. & 6,140 & 102 & May 20, 1950 & 22.49 & 20,000 & -- \\
\hline & $(05227500)$ & & & May 3, 1975 & -- & 14,500 & -- \\
\hline & & & & April 23, 1969 & -- & 14,400 & -- \\
\hline & & & & April 28, 2001 & 17.74 & 13,900 & -- \\
\hline & & & & April 26, 1965 & -- & 13,400 & -- \\
\hline \multirow[t]{5}{*}{3} & $\underline{\text { Mississippi River at Brainerd }}$ & 7,320 & 14 & April 30, 2001 & 16.70 & 17,800 & -- \\
\hline & $(05242300)$ & & & April 10, 1997 & 16.03 & 15,400 & -- \\
\hline & & & & April 30, 1996 & 14.59 & 14,000 & -- \\
\hline & & & & May 18, 1999 & 14.52 & 13,000 & -- \\
\hline & & & & July 10, 1993 & 13.65 & 12,200 & -- \\
\hline \multirow[t]{6}{*}{4} & Crow Wing River at Nimrod, Minn. & 1,030 & 73 & Oct. 10,1973 & 7.35 & 3,700 & $50-100$ \\
\hline & $(05244000)$ & & & April 18, 1979 & 7.24 & 3,610 & $50-100$ \\
\hline & & & & April 13, 1965 & ${ }^{4} 7.57$ & 2,890 & $10-25$ \\
\hline & & & & May 23, 1962 & 6.04 & 2,750 & $10-25$ \\
\hline & & & & June 9, 1968 & 6.07 & 2,750 & $10-25$ \\
\hline & & & & April 12, 2001 & 4.86 & $1,720(24$ th $)$ & $2-5$ \\
\hline \multirow[t]{5}{*}{5} & Long Prairie River at Long Prairie, Minn. & 434 & 30 & July 22, 1972 & 9.37 & 3,270 & $50-100$ \\
\hline & $(05245100)$ & & & April 9, 2001 & 8.81 & 2,660 & $25-50$ \\
\hline & & & & April 20, 1979 & 7.55 & 1,850 & $10-25$ \\
\hline & & & & April 8, 1997 & 7.90 & 1,580 & $5-10$ \\
\hline & & & & April 1, 1986 & 7.10 & 1,570 & $5-10$ \\
\hline \multirow[t]{5}{*}{6} & $\underline{\text { Crow Wing River near Pillager, Minn. }}$ & 3,520 & 33 & April 12, 2001 & 12.12 & 17,200 & -- \\
\hline & $(05247500)$ & & & April 12, 1969 & -- & 16,600 & -- \\
\hline & & & & April 7, 1997 & 11.73 & 16,300 & -- \\
\hline & & & & April 15, 1996 & 10.16 & 13,900 & -- \\
\hline & & & & April 14, 1974 & -- & 12,200 & -- \\
\hline \multirow[t]{6}{*}{7} & Sauk River near St. Cloud, Minn. & 1,030 & 67 & April 13, 1965 & 10.68 & 9,100 & $200-500$ \\
\hline & $(05270500)$ & & & April 13, 1951 & 7.89 & 5,580 & $25-50$ \\
\hline & & & & April 13, 1952 & 7.78 & 5,410 & $25-50$ \\
\hline & & & & April 11, 1969 & 7.96 & 5,300 & $25-50$ \\
\hline & & & & April 6, 1997 & 8.72 & 5,150 & $\sim 25$ \\
\hline & & & & April 14, 2001 & 8.37 & 4,780 (6th) & $10-25$ \\
\hline \multirow[t]{5}{*}{8} & $\underline{\text { Mississippi River at St. Cloud, Minn. }}$ & 13,320 & 13 & April 15, 2001 & 11.74 & 50,100 & -- \\
\hline & $(05270700)$ & & & April 8, 1997 & 11.44 & 46,900 & -- \\
\hline & & & & May 17, 1999 & 9.38 & 33,800 & -- \\
\hline & & & & April 22, 1996 & 8.70 & 27,300 & -- \\
\hline & & & & May 2, 1994 & 8.24 & 23,300 & -- \\
\hline \multirow[t]{6}{*}{9} & Elk River near Big Lake, Minn. & 559 & 75 & April 16, 1965 & 10.86 & 7,360 & $50-100$ \\
\hline & $(05275000)$ & & & April 10, 1969 & 10.08 & 5,980 & $25-50$ \\
\hline & & & & April 10, 1952 & 10.36 & 5,330 & $10-25$ \\
\hline & & & & May 7, 1912 & 10.10 & 5,180 & $10-25$ \\
\hline & & & & June 16, 1984 & 9.87 & 4,980 & $10-25$ \\
\hline & & & & April 27, 2001 & 9.46 & 4,520 (6th) & $10-25$ \\
\hline
\end{tabular}


Table 1. Provisional flood information on major Minnesota streams in the Mississippi River Basin. (Continued)

\begin{tabular}{|c|c|c|c|c|c|c|c|}
\hline $\begin{array}{l}\text { Station } \\
\text { number } \\
\text { (Figure 1) }\end{array}$ & $\begin{array}{l}\text { U.S. Geological Survey gaging station and } \\
\text { (downstream order number) }\end{array}$ & $\begin{array}{l}\text { Drainage } \\
\text { area } \\
\left(\mathrm{mi}^{2}\right)\end{array}$ & $\begin{array}{l}\text { Years of } \\
\text { peak flow } \\
\text { record }\end{array}$ & Date of flood & $\begin{array}{l}\text { Peak Stage } \\
\quad \text { (feet) }\end{array}$ & $\begin{array}{l}\text { Peak Discharge } \\
\qquad\left(\mathrm{ft}^{3} / \mathrm{s}\right)\end{array}$ & $\begin{array}{l}\text { Recurrence } \\
\text { interval } \\
\text { (years) }\end{array}$ \\
\hline \multirow[t]{5}{*}{10} & \multirow{5}{*}{$\begin{array}{l}\text { Crow River at Rockford, Minn. } \\
(05280000)\end{array}$} & \multirow[t]{5}{*}{2,640} & \multirow[t]{5}{*}{80} & April 16, 1965 & 19.27 & 22,400 & $100-200$ \\
\hline & & & & April 13, 1969 & 16.51 & 15,100 & $25-50$ \\
\hline & & & & April 13, 1952 & 16.24 & 13,900 & $\sim 25$ \\
\hline & & & & June 26, 1957 & 16.14 & 13,500 & $10-25$ \\
\hline & & & & April 16, 2001 & 14.50 & 13,400 & $10-25$ \\
\hline \multirow[t]{5}{*}{11} & Rum River near St. Francis, Minn. & \multirow[t]{5}{*}{1,360} & \multirow[t]{5}{*}{72} & April 20, 1965 & 11.57 & 10,100 & $10-25$ \\
\hline & \multirow[t]{4}{*}{$(05286000)$} & & & April 13, 1969 & 11.63 & 10,100 & $10-25$ \\
\hline & & & & July 29, 1972 & 11.20 & 9,540 & $10-25$ \\
\hline & & & & April 13, 1952 & 11.03 & 9,260 & $10-25$ \\
\hline & & & & April 28, 2001 & 10.47 & 8,500 & $10-25$ \\
\hline \multirow[t]{5}{*}{12} & Elm Creek near Champlin, Minn. & \multirow[t]{5}{*}{86} & \multirow[t]{5}{*}{23} & April 25, 2001 & 10.03 & 880 & $10-25$ \\
\hline & \multirow[t]{4}{*}{$(05287890)$} & & & Mar. 27, 1986 & 9.93 & 812 & $10-25$ \\
\hline & & & & April 30, 1994 & 9.69 & 669 & $5-10$ \\
\hline & & & & Mar. 18, 1985 & 9.50 & 579 & $5-10$ \\
\hline & & & & May 15, 1999 & 9.40 & 538 & $2-5$ \\
\hline \multirow[t]{6}{*}{13} & \multirow{6}{*}{$\begin{array}{l}\text { Mississippi River at Anoka, Minn. } \\
(05288500)\end{array}$} & \multirow[t]{5}{*}{19,100} & \multirow[t]{5}{*}{71} & April 17, 1965 & 19.53 & 91,000 & $100-200$ \\
\hline & & & & April 14, 1952 & 17.51 & 75,900 & $25-50$ \\
\hline & & & & April 14, 1969 & 16.84 & 72,500 & $25-50$ \\
\hline & & & & April 9, 1997 & 16.44 & 69,800 & $25-50$ \\
\hline & & & & April 15, 2001 & 15.77 & 65,600 & $10-25$ \\
\hline & & \multicolumn{3}{|c|}{ Minnesota River Basin } & & & \\
\hline \multirow[t]{5}{*}{14} & \multirow{5}{*}{$\begin{array}{l}\text { Little Minnesota River near Peever, S. Dak } \\
(05290000)\end{array}$} & 438 & 54 & July 25,1993 & 13.58 & 8,900 & $50-100$ \\
\hline & & & & April 8, 1952 & 12.16 & 4,730 & $10-25$ \\
\hline & & & & Mar. 25, 1943 & ${ }^{4} 13.35$ & 4,320 & $10-25$ \\
\hline & & & & Mar. 28, 1997 & 8.82 & 3,590 & $10-25$ \\
\hline & & & & April 12, 2001 & 8.64 & 3,510 & $10-25$ \\
\hline 15 & $\underline{\text { Whetstone River near Big Stone City, S. Dak }}$ & 398 & 75 & April 8, 2001 & 15.93 & 9,980 & $25-50$ \\
\hline & $(05291000)$ & & & April 6, 1997 & 14.21 & 7,940 & $10-25$ \\
\hline & & & & April 8, 1969 & 14.32 & 6,870 & $10-25$ \\
\hline & & & & April 8, 1952 & 13.64 & 5,710 & $10-25$ \\
\hline & & & & April 11, 1947 & 13.95 & 5,500 & $10-25$ \\
\hline 16 & Minnesota River at Ortonville, Minn. & 1,160 & 64 & April 10, 1997 & 12.85 & 5,070 & -- \\
\hline & (05292000) & & & April 14, 2001 & 12.06 & 4,850 & -- \\
\hline & & & & April 13, 1952 & 12.92 & 3,060 & -- \\
\hline & & & & July 28, 1993 & 9.99 & 2,950 & -- \\
\hline & & & & Mar. 27, 1989 & 10.76 & ${ }^{5} 2,780$ & -- \\
\hline 17 & $\underline{\text { North Fork Yellow Bank River near Odessa, S. Dak }}$ & 208 & 11 & April 8, 2001 & 16.84 & 7,300 & -- \\
\hline & $(05292704)$ & & & Mar. 31, 1997 & 15.94 & 4,670 & -- \\
\hline & & & & July 8, 1994 & 14.62 & 2,580 & -- \\
\hline & & & & April 19, 1995 & 14.15 & 2,420 & -- \\
\hline & & & & June 18,1992 & 13.32 & 2,020 & -- \\
\hline 18 & $\underline{\text { Minnesota River near Lac qui Parle, Minn. }}$ & 4,050 & 55 & April 14, 2001 & ${ }^{6} 40.05$ & 30,000 & -- \\
\hline & $(05301000)$ & & & April 12, 1969 & 39.75 & 29,400 & - \\
\hline & & & & April 10, 1952 & 37.98 & ${ }^{7} 19,700$ & -- \\
\hline & & & & April 8, 1986 & 36.80 & ${ }^{7} 13,200$ & -- \\
\hline & & & & April 14, 1965 & 36.17 & ${ }^{7} 10,700$ & -- \\
\hline 19 & Chippewa River near Milan, Minn. & 1,880 & 65 & April 6, 1997 & 18.03 & 14,400 & $50-100$ \\
\hline & $(05304500)$ & & & April 10, 2001 & 14.63 & 11,600 & $25-50$ \\
\hline & & & & April 9, 1969 & ${ }^{8} 15.45$ & 11,400 & $25-50$ \\
\hline & & & & July 6, 1995 & ${ }^{8} 13.48$ & 8,440 & $10-25$ \\
\hline & & & & April 6, 1952 & ${ }^{8} 12.12$ & 6,930 & $10-25$ \\
\hline
\end{tabular}


Table 1. Provisional flood information on major Minnesota streams in the Mississippi River Basin. (Continued)

\begin{tabular}{|c|c|c|c|c|c|c|c|}
\hline $\begin{array}{c}\text { Station } \\
\text { number } \\
\text { (Figure 1) }\end{array}$ & $\begin{array}{l}\text { U.S. Geological Survey gaging station and } \\
\text { (downstream order number) }\end{array}$ & $\begin{array}{c}\text { Drainage } \\
\text { area } \\
\left(\mathrm{mi}^{2}\right)\end{array}$ & $\begin{array}{c}\text { Years of } \\
\text { peak flow } \\
\text { record }\end{array}$ & Date of flood & $\begin{array}{l}\text { Peak Stage } \\
\quad(\text { feet })\end{array}$ & $\begin{array}{l}\text { Peak Discharge } \\
\quad\left(\mathrm{ft}^{3} / \mathrm{s}\right)\end{array}$ & $\begin{array}{c}\text { Recurrence } \\
\text { interval } \\
\text { (years) }\end{array}$ \\
\hline \multirow[t]{5}{*}{20} & \multirow{5}{*}{$\begin{array}{l}\text { Minnesota River at Montevideo, Minn. } \\
(05311000)\end{array}$} & \multirow[t]{3}{*}{6,180} & \multirow[t]{3}{*}{92} & April 6, 1997 & 23.90 & 47,500 & $100-200$ \\
\hline & & & & April 12, 1969 & 21.68 & 35,100 & $50-100$ \\
\hline & & & & April 13, 2001 & ${ }^{9} 22.15$ & 33,800 & $50-100$ \\
\hline & & & & April 10, 1952 & 20.02 & 24,500 & $25-50$ \\
\hline & & & & June 25,1919 & 17.45 & 22,000 & $\sim 25$ \\
\hline \multirow[t]{6}{*}{21} & \multirow{6}{*}{$\begin{array}{l}\text { Yellow Medicine River near Granite Falls, Minn. } \\
(05313500)\end{array}$} & \multirow[t]{6}{*}{664} & \multirow[t]{6}{*}{71} & April 10, 1969 & 14.90 & 17,200 & $100-200$ \\
\hline & & & & June 18, 1957 & 12.41 & 11,800 & $50-100$ \\
\hline & & & & April 9, 1952 & 10.50 & 9,610 & $25-50$ \\
\hline & & & & Mar. 31, 1997 & 11.80 & 9,020 & $25-50$ \\
\hline & & & & June 21, 1993 & 11.46 & 8,380 & $10-25$ \\
\hline & & & & April 26, 2001 & 8.00 & 3,860 (10th) & $5-10$ \\
\hline \multirow[t]{5}{*}{22} & \multirow{5}{*}{$\begin{array}{l}\text { Redwood River near Marshall, Minn. } \\
(05315000)\end{array}$} & \multirow[t]{5}{*}{259} & \multirow[t]{5}{*}{62} & May 9, 1993 & 17.00 & 6,380 & $50-100$ \\
\hline & & & & April 10, 1969 & -- & 5,590 & $\sim 50$ \\
\hline & & & & June 17,1957 & ${ }^{8} 10.14$ & 5,370 & $25-50$ \\
\hline & & & & April 24, 2001 & 15.65 & 3,930 & $10-25$ \\
\hline & & & & Mar. 30, 1997 & 15.82 & 3,310 & $10-25$ \\
\hline \multirow[t]{6}{*}{23} & \multirow{6}{*}{$\begin{array}{l}\text { Redwood River near Redwood Falls, Minn. } \\
(05316500)\end{array}$} & \multirow[t]{6}{*}{629} & \multirow[t]{6}{*}{76} & June 18, 1957 & 15.92 & 19,700 & $100-200$ \\
\hline & & & & April 9, 1969 & 14.58 & 14,100 & $50-100$ \\
\hline & & & & June 18, 1993 & 15.73 & 12,600 & $25-50$ \\
\hline & & & & Mar. 31, 1960 & 10.95 & 7,660 & $10-25$ \\
\hline & & & & Mar. 30, 1997 & 11.84 & ${ }^{3} 7,200$ & $10-25$ \\
\hline & & & & April 25, 2001 & 10.93 & 5,830 (8th) & $10-25$ \\
\hline \multirow[t]{5}{*}{24} & \multirow{5}{*}{$\begin{array}{l}\text { Cottonwood River near New Ulm, Minn. } \\
(05317000)\end{array}$} & 1,300 & 75 & April 10, 1969 & 19.15 & 28,700 & $\sim 100$ \\
\hline & & & & April 8, 1965 & ${ }^{3} 20.86$ & 26,000 & $50-100$ \\
\hline & & & & June 19,1993 & 18.90 & 24,300 & $50-100$ \\
\hline & & & & April 25, 2001 & 18.18 & 20,200 & $25-50$ \\
\hline & & & & Mar. 30, 1962 & 15.88 & 14,200 & $10-25$ \\
\hline 25 & Little Cottonwood River near Courtland, Minn. & 170 & 28 & June 20, 1993 & ${ }^{10} 10.45$ & 3,520 & $100-200$ \\
\hline & $(05317200)$ & & & April 5, 2001 & 9.31 & 2,010 & $10-25$ \\
\hline & & & & June 17, 1996 & ${ }^{11} 8.02$ & 1,470 & $5-10$ \\
\hline & & & & Mar. 16, 1985 & 8.96 & 1,340 & $5-10$ \\
\hline & & & & Mar. 22, 1986 & 8.56 & 1,060 & $2-5$ \\
\hline 26 & Watonwan River near Garden City, Minn. & 851 & 31 & June 20, 1993 & 1215.91 & 13,900 & $50-100$ \\
\hline & $(05319500)$ & & & April 15, 2001 & 12.28 & 7,400 & $\sim 10$ \\
\hline & & & & May 21, 1944 & 9.84 & 5,620 & $5-10$ \\
\hline & & & & June 2, 1980 & 9.80 & 5,250 & $5-10$ \\
\hline & & & & June 20, 1996 & 9.44 & 4,820 & $2-5$ \\
\hline 27 & Blue Earth River near Rapidan, Minn. & 2,410 & 89 & April 9, 1965 & 21.36 & 43,100 & -- \\
\hline & $(05320000)$ & & & April 8, 1951 & 14.97 & 26,100 & -- \\
\hline & & & & April 10, 1969 & 13.54 & 21,100 & -- \\
\hline & & & & June 20, 1993 & 13.32 & 20,300 & -- \\
\hline & & & & June 9, 1953 & 12.91 & 19,700 & -- \\
\hline & & & & April 14, 2001 & 12.04 & 17,200 (6th) & -- \\
\hline 28 & Le Sueur River near Rapidan, Minn. & 1,110 & 59 & April 8, 1965 & 22.10 & 24,700 & $\sim 100$ \\
\hline & $(05320500)$ & & & May 22,1960 & 22.72 & 21,200 & $50-100$ \\
\hline & & & & June 16, 2001 & 13.80 & 14,600 & $10-25$ \\
\hline & & & & April 7, 1951 & 19.73 & 13,200 & $10-25$ \\
\hline & & & & Feb. 28, 1992 & ${ }^{4} 14.18$ & 12,600 & $10-25$ \\
\hline 29 & Minnesota River at Mankato, Minn. & 14,900 & 99 & April 10, 1965 & ${ }^{8} 29.09$ & 94,100 & $100-200$ \\
\hline & $(05325000)$ & & & April 10, 1997 & 27.61 & 79,800 & $50-100$ \\
\hline & & & & April 12, 1969 & $8_{27.07}$ & 76,700 & $50-100$ \\
\hline & & & & June 21, 1993 & 30.11 & 75,600 & $50-100$ \\
\hline & & & & April 16, 2001 & 26.96 & 73,700 & $25-50$ \\
\hline
\end{tabular}


Table 1. Provisional flood information on major Minnesota streams in the Mississippi River Basin. (Continued)

\begin{tabular}{|c|c|c|c|c|c|c|c|}
\hline $\begin{array}{c}\text { Station } \\
\text { number } \\
\text { (Figure 1) }\end{array}$ & $\begin{array}{l}\text { U.S. Geological Survey gaging station and } \\
\text { (downstream order number) }\end{array}$ & $\begin{array}{c}\text { Drainage } \\
\text { area } \\
\left(\mathrm{mi}^{2}\right)\end{array}$ & $\begin{array}{c}\text { Years of } \\
\text { peak flow } \\
\text { record }\end{array}$ & Date of flood & $\begin{array}{l}\text { Peak Stage } \\
\quad(\text { feet })\end{array}$ & $\begin{array}{l}\text { Peak Discharge } \\
\left(\mathrm{ft}^{3} / \mathrm{s}\right)\end{array}$ & $\begin{array}{c}\text { Recurrence } \\
\text { interval } \\
\text { (years) }\end{array}$ \\
\hline \multirow[t]{5}{*}{30} & \multirow{5}{*}{$\begin{array}{l}\text { High Island Creek near Henderson, Minn. } \\
(05327000)\end{array}$} & \multirow[t]{5}{*}{238} & \multirow[t]{5}{*}{27} & July 25, 1997 & 9.31 & 2,830 & $10-25$ \\
\hline & & & & June 17,1993 & 9.72 & 2,750 & $10-25$ \\
\hline & & & & April 18, 2001 & ${ }^{10} 9.81$ & 2,200 & $5-10$ \\
\hline & & & & Sep. 15, 1992 & 8.32 & 1,770 & $2-5$ \\
\hline & & & & Aug. 25, 1981 & 9.09 & 1,760 & $2-5$ \\
\hline \multirow[t]{5}{*}{31} & \multirow{6}{*}{$\begin{array}{l}\text { Minnesota River near Jordan, Minn. } \\
(05330000)\end{array}$} & \multirow[t]{5}{*}{16,200} & \multirow[t]{5}{*}{67} & April 11, 1965 & -- & ${ }^{8} 117,000$ & $\sim 100$ \\
\hline & & & & June 24, 1993 & 33.52 & 92,200 & $25-50$ \\
\hline & & & & April 18, 2001 & 33.11 & 87,100 & $25-50$ \\
\hline & & & & April 14, 1969 & 32.85 & 84,600 & $25-50$ \\
\hline & & & & April 12, 1997 & 32.24 & 82,300 & $25-50$ \\
\hline \multicolumn{7}{|c|}{ Mississippi River Basin } & \\
\hline \multirow[t]{5}{*}{32} & \multirow{5}{*}{$\begin{array}{l}\text { Mississippi River at St. Paul, Minn. } \\
(05331000)\end{array}$} & \multirow[t]{5}{*}{36,800} & \multirow{5}{*}{134} & April 16, 1965 & ${ }^{8} 26.01$ & 171,000 & $200-500$ \\
\hline & & & & April 15, 1969 & ${ }^{8} 24.52$ & 156,000 & $100-200$ \\
\hline & & & & April 18, 2001 & 23.46 & 140,000 & $50-100$ \\
\hline & & & & April 13, 1997 & ${ }^{8} 22.37$ & 134,000 & $50-100$ \\
\hline & & & & April 16, 1952 & $8_{22.02}$ & 125,000 & $25-50$ \\
\hline \multicolumn{8}{|c|}{ St. Croix River Basin } \\
\hline \multirow[t]{5}{*}{33} & \multirow{5}{*}{$\begin{array}{l}\text { Kettle River below Sandstone, Minn. } \\
(05336700)\end{array}$} & \multirow[t]{5}{*}{868} & \multirow[t]{5}{*}{35} & July 23, 1972 & 15.38 & 17,200 & $25-50$ \\
\hline & & & & April 24, 2001 & 14.67 & 15,600 & $10-25$ \\
\hline & & & & April 21, 1979 & 13.40 & 13,700 & $10-25$ \\
\hline & & & & April 1965 & 12.96 & 13,400 & $10-25$ \\
\hline & & & & April 12, 1971 & 12.36 & 12,100 & $5-10$ \\
\hline 34 & $\underline{\text { Knife River near Mora, Minn. }}$ & 102 & 27 & April 23, 2001 & 7.76 & 3,450 & $25-50$ \\
\hline & $(05337400)$ & & & April 6, 1997 & 6.48 & 1,870 & $5-10$ \\
\hline & & & & May 10, 1979 & 6.31 & 1,840 & $5-10$ \\
\hline & & & & April 1, 1986 & 6.22 & 1,780 & $5-10$ \\
\hline & & & & Aug. 27, 1995 & 6.00 & 1,580 & $\sim 5$ \\
\hline 35 & Snake River near Pine City, Minn. & 974 & 51 & July 27, 1972 & 10.38 & 14,300 & $25-50$ \\
\hline & $(05338500)$ & & & May 9, 1950 & -- & 12,500 & $10-25$ \\
\hline & & & & April 18, 1965 & 9.56 & 11,500 & $10-25$ \\
\hline & & & & April 27, 2001 & 9.23 & 11,000 & $10-25$ \\
\hline & & & & April 12, 1969 & 9.08 & 10,200 & $5-10$ \\
\hline 36 & $\underline{\text { St. Croix River at St. Croix Falls, WI }}$ & 6,240 & 96 & April 25, 2001 & 25.88 & 60,900 & $50-100$ \\
\hline & $(05340500)$ & & & May 8, 1950 & 25.19 & 54,900 & $25-50$ \\
\hline & & & & April 18, 1965 & 20.98 & 45,700 & $10-25$ \\
\hline & & & & Mar. 20, 1945 & 20.53 & 44,600 & $10-25$ \\
\hline & & & & May 4, 1954 & 20.40 & 44,400 & $10-25$ \\
\hline & & Iississippi 1 & River Basi & & & & \\
\hline 37 & Mississippi River at Prescott, WI & 44,800 & 73 & April 18, 1965 & 43.11 & 228,000 & $100-200$ \\
\hline & $(05344500)$ & & & April 16, 1969 & 41.48 & 199,000 & $50-100$ \\
\hline & & & & April 16, 2001 & 40.35 & 178,000 & $25-50$ \\
\hline & & & & April 12, 1997 & 40.09 & 161,000 & $25-50$ \\
\hline & & & & April 16, 1952 & 39.03 & 155,000 & $\sim 25$ \\
\hline 38 & Vermillion River near Empire, Minn. & 129 & 33 & Sep. 16,1992 & 10.00 & 6,570 & $25-50$ \\
\hline & $(05345000)$ & & & April 1965 & 7.50 & 6,200 & $25-50$ \\
\hline & & & & June 27,1998 & 9.17 & 3,490 & $10-25$ \\
\hline & & & & Sep. 18,1942 & 6.00 & 2,030 & $5-10$ \\
\hline & & & & June 18,1993 & 8.37 & 1,780 & $\sim 5$ \\
\hline & & & & April 8, 2001 & 6.81 & 677 (14th) & $1-2$ \\
\hline 39 & Straight River near Faribault, Minn. & 435 & 36 & July 7, 1990 & 11.31 & 6,030 & $10-25$ \\
\hline & $(05353800)$ & & & April 13, 2001 & 10.73 & 6,010 & $10-25$ \\
\hline & & & & May 1, 1990 & 11.20 & 5,990 & $10-25$ \\
\hline & & & & July 1,1983 & 11.11 & 5,840 & $10-25$ \\
\hline & & & & June 17,1993 & 11.16 & 5,730 & $10-25$ \\
\hline
\end{tabular}


Table 1. Provisional flood information on major Minnesota streams in the Mississippi River Basin. (Continued)

\begin{tabular}{|c|c|c|c|c|c|c|c|}
\hline $\begin{array}{c}\text { Station } \\
\text { number } \\
\text { (Figure 1) }\end{array}$ & $\begin{array}{l}\text { U.S. Geological Survey gaging station and } \\
\text { (downstream order number) }\end{array}$ & $\begin{array}{c}\text { Drainage } \\
\text { area } \\
\left(\mathrm{mi}^{2}\right)\end{array}$ & $\begin{array}{c}\text { Years of } \\
\text { peak flow } \\
\text { record }\end{array}$ & Date of flood & $\begin{array}{l}\text { Peak Stage } \\
\quad \text { (feet) }\end{array}$ & $\begin{array}{l}\text { Peak Discharge } \\
\left(\mathrm{ft}^{3} / \mathrm{s}\right)\end{array}$ & $\begin{array}{c}\text { Recurrence } \\
\text { interval } \\
\text { (years) }\end{array}$ \\
\hline \multirow[t]{6}{*}{40} & \multirow{6}{*}{$\begin{array}{l}\text { Cannon River at Welch, Minn. } \\
(05355200)\end{array}$} & \multirow[t]{6}{*}{1,340} & \multirow[t]{6}{*}{70} & April 8, 1965 & 14.01 & 36,100 & $200-500$ \\
\hline & & & & June 27, 1998 & 15.05 & 23,500 & $25-50$ \\
\hline & & & & Feb. 10, 1966 & ${ }^{4} 13.93$ & 20,800 & $25-50$ \\
\hline & & & & Sep. 22, 1986 & 13.80 & 18,900 & $10-25$ \\
\hline & & & & June 17,1993 & 13.19 & 17,200 & $10-25$ \\
\hline & & & & April 12, 2001 & 11.43 & 11,900 (9th) & $5-10$ \\
\hline \multirow[t]{6}{*}{41} & \multirow{6}{*}{$\begin{array}{l}\text { South Fork Zumbro River at Rochester, Minn. } \\
\text { (05372995) }\end{array}$} & \multirow[t]{6}{*}{303} & \multirow[t]{6}{*}{51} & July 6, 1978 & ${ }^{8} 23.36$ & 30,500 & $100-200$ \\
\hline & & & & Mar. 1, 1965 & ${ }^{8} 19.12$ & 19,600 & $25-50$ \\
\hline & & & & Mar. 29, 1962 & ${ }^{8} 18.46$ & 18,000 & $25-50$ \\
\hline & & & & July 21, 1951 & ${ }^{8} 17.50$ & 15,000 & $25-50$ \\
\hline & & & & Mar. 26, 1961 & ${ }^{8} 15.43$ & 10,900 & $10-25$ \\
\hline & & & & April 12, 2001 & 13.68 & 5220 (17th) & $2-5$ \\
\hline \multirow[t]{5}{*}{42} & Mississippi River at Winona, Minn. & \multirow[t]{5}{*}{59,200} & \multirow[t]{5}{*}{122} & April 19, 1965 & 20.77 & 268,000 & $200-500$ \\
\hline & \multirow[t]{4}{*}{$(05378500)$} & & & April 17, 2001 & 20.07 & 237,000 & $\sim 100$ \\
\hline & & & & April 19, 1969 & 19.44 & 218,000 & $50-100$ \\
\hline & & & & April 11, 1997 & 18.27 & 194,000 & $25-50$ \\
\hline & & & & April 20, 1952 & 17.91 & 190,000 & $\sim 25$ \\
\hline \multirow[t]{7}{*}{43} & \multirow{7}{*}{$\begin{array}{l}\text { Cedar River near Austin, Minn. } \\
(05457000)\end{array}$} & \multirow[t]{6}{*}{399} & \multirow[t]{6}{*}{62} & July 10, 2000 & 21.49 & 15,300 & $100-200$ \\
\hline & & & & July 17, 1978 & 20.35 & 12,400 & $25-50$ \\
\hline & & & & Aug. 15, 1993 & 19.43 & 10,800 & $10-25$ \\
\hline & & & & Mar. 29, 1962 & 17.18 & 9,530 & $10-25$ \\
\hline & & & & Mar. 26, 1961 & 17.03 & 9,400 & $10-25$ \\
\hline & & & & April 12, 2001 & 15.77 & $7,710(11$ th $)$ & $5-10$ \\
\hline & & \multicolumn{3}{|c|}{ Des Moines River Basin } & & & \\
\hline \multirow[t]{6}{*}{44} & \multirow{6}{*}{$\begin{array}{l}\text { Des Moines River at Jackson, Minn. } \\
(05476000)\end{array}$} & \multirow[t]{6}{*}{1,250} & 76 & April 11, 1969 & 19.45 & 15,700 & $\sim 200$ \\
\hline & & & & April 9, 1965 & -- & 9,530 & $25-50$ \\
\hline & & & & June 8,1953 & 17.43 & 8,360 & $25-50$ \\
\hline & & & & July 7, 1993 & 16.67 & 8,250 & $25-50$ \\
\hline & & & & April 13, 1984 & 16.31 & 7,770 & $10-25$ \\
\hline & & & & April 28, 2001 & 15.47 & 6,590 (6th) & $10-25$ \\
\hline
\end{tabular}

${ }^{1}$ Result of dam failure.

2 Daily-mean discharge

${ }^{3}$ Reached a higher discharge on Nov. 11, 2000; discharge, 2,980 $\mathrm{ft}^{3} / \mathrm{s}$.

${ }^{4}$ Backwater from ice.

${ }^{5}$ Estimated value.

${ }^{6}$ Flood of April 7, 1997 reached a stage of $41.66 \mathrm{ft}$ (information from the U.S. Army Corp of Engineers)

${ }^{7}$ Affected to an unknown degree by regulation.

${ }^{8}$ Site then in use.

${ }^{9}$ Gage height from U.S. Army Corp of Engineers.

${ }^{10}$ Possible backwater from the Minnesota River.

${ }^{11}$ Maximum recorded, was probably higher.

12 Maximum stage and discharge outside the period of record: April 7, 1965, stage, $18.89 \mathrm{ft}$; discharge, $19,000 \mathrm{ft}^{3} / \mathrm{s}$. Historic peaks used in floodfrequency analysis. 


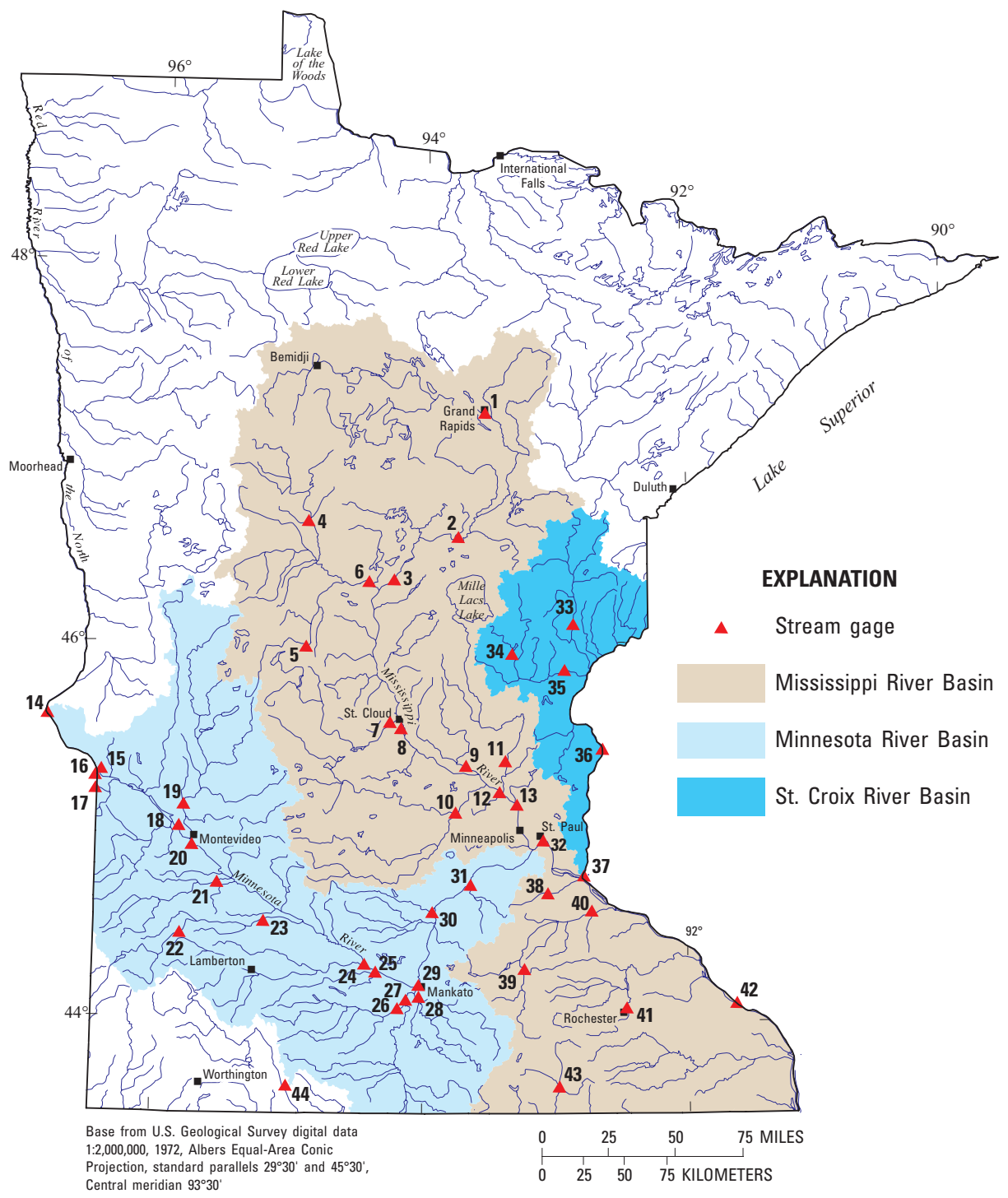

Figure 1. Location of stream-gaging stations.

depending on the magnitude of these additional flows. This is a primary reason why flood frequencies are not included in this report for stations with less than 20 years of record. Even with 20 or more years of record, significant changes to previously established flood frequencies are possible.

This report presents preliminary water-resources 2001 flood data obtained from streamflow-gaging stations in the Mississippi River Basin (fig. 1).

\section{CLIMATIC CONDITIONS}

Above normal rains in early November 2000 followed by snowfalls later in the month resulted in precipitation totals that were well above historical Minnesota (Minnesota State Climatology Office, electronic commun., accessed
July 16, 2001, URL

http://climate.umn.edu/doc/journal/flood_ 2001/flood_2001.htm). With additional snowfall throughout the winter, total accumulation in parts of southern Minnesota was 18 to 24 inches greater than for a normal winter, placing total snowfall in the 90 to 95 percentiles. Typically, the snow pack would lose much of its' water equivalence from late winter to early spring, before the arrival of spring rains. However, below-normal temperatures for February and March delayed the snowmelt and only compacted the existing snow cover. Snow-to-water equivalents in early March ranged from 3 to 5 inches and by the end of March had changed little. In April, heavy rains fell over much of the central and southern parts of the State.

Precipitation totals for the month were greater than 6 inches in some areas, which was greater than 4 inches above normal. This rainfall, along with the greater than normal snow-to-water equivalents, provided the runoff that resulted in spring flooding. These climatic conditions accounted for most of the flooding in Minnesota during spring 2001. Depth of ground frost, which was minimized by early snowfalls that insulated the ground from the cold winter air, was minimal in many parts of southern Minnesota that otherwise had most of the conditions that can lead to serious flood potential.

\section{CHRONOLOGY OF FLOODING}

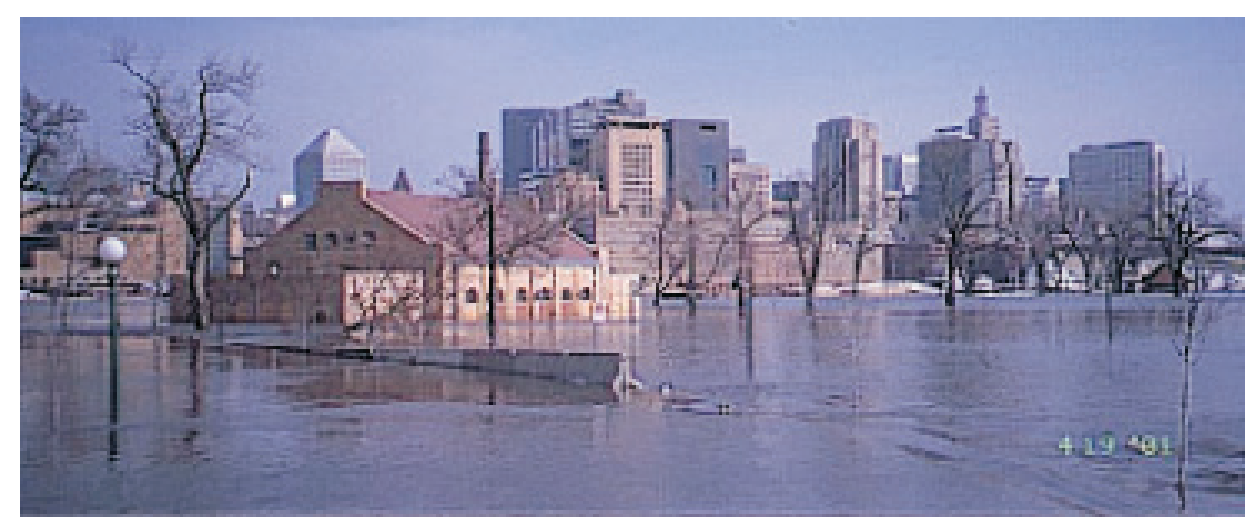

Mississippi River at Harriet Island, downtown St. Paul, Minn., April 19, 2001. (U.S. Geological Survey)
By the first week of April, tributaries to the Minnesota River, Mississippi River, 
and St. Croix Rivers were on the rise.

Rainfall amounts ranging from about 1.0 inch to greater than 2.0 inches fell in westcentral Minnesota and east-central South Dakota on April 6th and 7th. On April 8th the Whetstone River near Big Stone City, South Dakota (station number 15, table 1) crested at a stage of 15.93 feet while the North Fork of the Yellow Bank River near Odessa (station number 17, table 1) crested at 16.84 feet. Both crests were records for the period of gage operation. The recurrence interval of the peak discharge on the Whetstone River was between 25-year and 50-years. Other tributaries to the Minnesota River, as well as the Minnesota River at and upstream of Montevideo, were cresting by the second week of April.

Record flows were recorded on the Crow Wing River near Pillager (April 12, station number 6, table1), Minnesota River near Lac qui Parle (April 14th, station

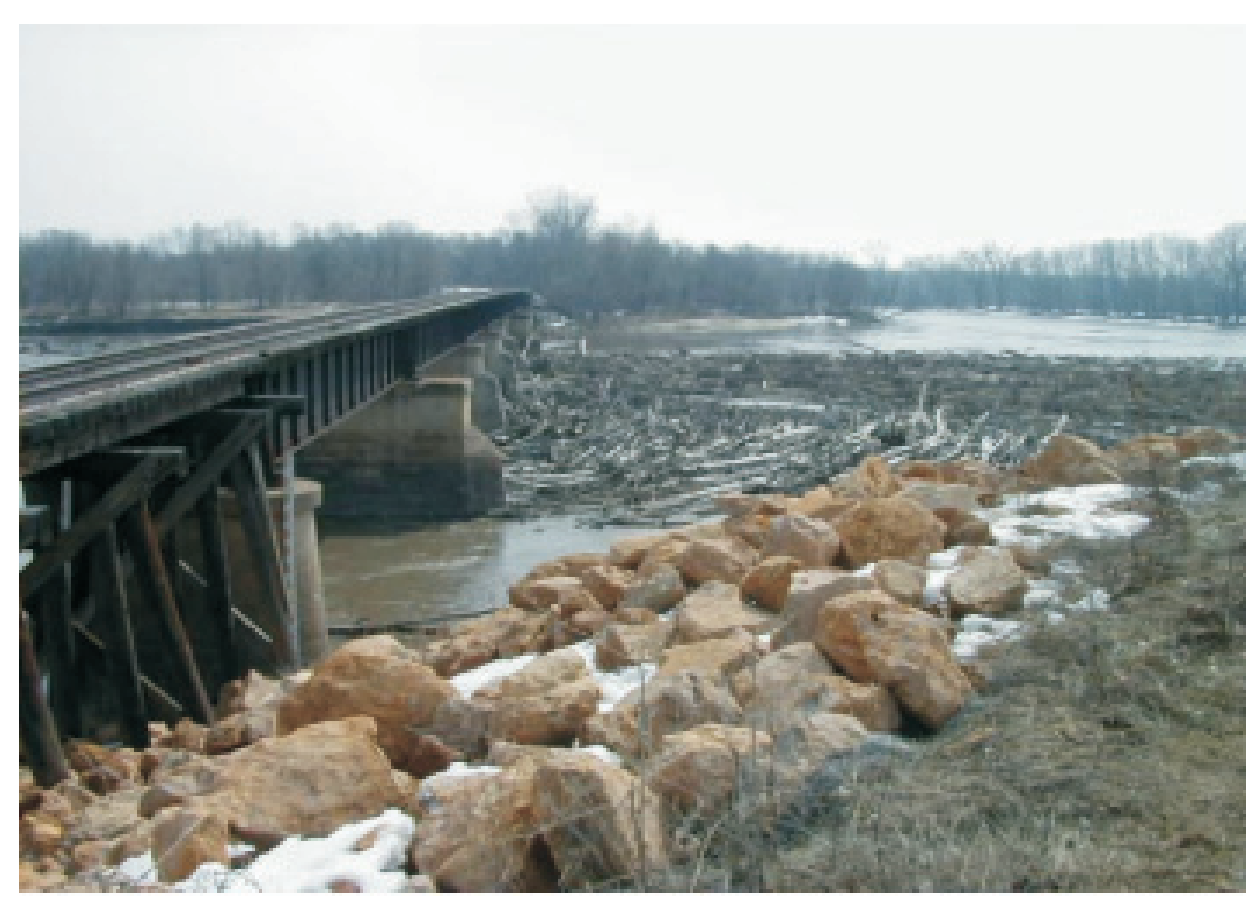

number18, table 1), and Mississippi River at St. Cloud (April 15th, station number 8, table 1), and Elm Creek near Champlin, Minn. (April 25, station number 12, table 1). These three sites are influenced by regulation and thus no recurrence intervals were computed. By comparison, the Minnesota River at Montevideo (station number 20, table 1) reached its' third highest discharge of record on April 13. This flow had a recurrence interval of between 50 and 100 years. On April 23rd the flooding was aggravated and prolonged when a storm produced greater than two inches of rain throughout much of Minnesota, and in some localities, greater than 3 inches (Minnesota State Climatology Office, electronic comm., accessed Aug. 15, 2001, URL http://climate.umn.edu/doc/journal/april2 001.htm). The cities of Lamberton, St. Cloud, and Worthington all reported greater than 3.5 inches of rain. Rivers

Log jam on the Minnesota River at Carver, Minn., April 25, 2001. (Minnesota Department of Public Safety, Emergency Management)

tributary to the St. Croix as well as the St. Croix River itself crested towards the end of April. Record flows were recorded at the Knife River near Mora (April 23rd, station number 34, table 1) and the St. Croix River at St. Croix Falls, Wisconsin (April 25th, station number 36, table 1). The peak flow at St. Croix River at St. Croix Falls had a recurrence interval of 50-100 years, while the recurrence intervals for the peak flows on the Knife and Kettle (station number 33 , table 1) Rivers were in the 25-50 and 10-25 year range, respectively.

In summary, four stream-gaging stations recorded peak flows between the 50-year and 100-year recurrence interval: the Minnesota River at Montevideo; Mississippi River at St. Paul; St. Croix River at St. Croix Falls, Wisconsin; and Mississippi River at Winona. Six stations with at least 20 years of record had record peak flows. These include the Crow Wing River near Pillager; Elm Creek near Champlin; Whetstone River near Big Stone City, South Dakota; Minnesota River near Lac qui Parle; Knife River near Mora; and St. Croix River at St. Croix Falls, Wisconsin.

The USGS wishes to acknowledge the support and cooperation of these agencies that assisted with the collection of field data: U.S. Army Corps of Engineers, Minnesota Department of Natural Resources, and National Weather Service.

G.B. Mitton, Hydrologist
For water information:

District Chief

2280 Woodale Drive

Mounds View, MN 55112

(763)783-3100

\section{For more information contact any of the following:}

For more information on all USGS reports and products (including maps, images, and computerized data), call 1-888-ASK-USGS
Addtional earth sciences information can be found by accessing the USGS "Home Page" on the World Wide Web at "http://www.usgs.gov". 